\title{
Joe Selby
}

As part of the Affordable Care Act of 2010, the US Congress created the Patient-Centered Outcomes Research Institute (PCORI). Just over a year since the creation of the comparative effectiveness research (CER) organization, the PCORI has now appointed its first Executive Director, Joe Selby. A physician who formerly directed research at Kaiser Permanente, northern California, Selby will now supervise the formation of the nascent institute's plan of action. And by 2014 he will be overseeing an expected annual research budget of US\$500 million. Speaking with Asher Mullard, Selby explained the case and agenda for the PCORI.

\section{Q How did the formation of the PCORI affect the CER landscape? \\ It was a pretty big incremental change. The Agency for Healthcare Research and Quality (AHRQ) had already been focusing on CER for several years, particularly through their Developing Evidence to Inform Decisions about Effectiveness (DEcIDE) programme. A branch of that has focused on developing the methods of CER, and they have funded some good CER studies. The US National Institutes of Health (NIH) has also been funding some CER clinical trials, a couple of which were landmarks. And I think they both deserve credit for being in the ball game early on. But to create a body dedicated to CER was a pretty big incremental advance.}

\section{Q How will you differentiate yourself from the AHRQ and the NIH?}

We have the heads of the AHRQ and the $\mathrm{NIH}$ on our governing board, and I think that will hopefully help to ensure we are not redundant. It's nice to have them in the room as we're planning our research agenda. And I hope that the commitment of these two bodies to CER will hopefully increase, even with the formation of the PCORI.

Our name gives you a bit of a hint of the different direction we will be taking though: we're not called the 'comparative effectiveness research' institute, we're called the 'Patient-Centered Outcomes Research (PCOR)' Institute. We will be distinguishing ourselves by being the most patient-centric institute that has ever existed, making sure that our research incorporates the principles of 'patient-centredness' while simultaneously not overlooking the relevance of engaging providers, physicians and others in the health-care community.
Q How much of this focus on PCOR, rather than CER, is merely semantics?

That's a good question, in part because no one quite knows what difference being patient-centred will really make yet. You're just going to have to stay tuned, I guess. But we will certainly be doing CER - that's what is in the legislation.

\section{Q Will cost-effectiveness analyses fit into your work?}

My sense - and it's just a sense because I've not discussed it much with the rest of the board - is that we don't see ourselves funding cost-effectiveness analyses. We also don't anticipate funding studies that generate quality-adjusted life years (QALYs), which link costs to outcomes. Arguably there is some subjectivity in QALYs, and it's hard not to get into difficult questions such as whether a year of life saved between $40-50$ years of age is equivalent to a year of life saved between $80-90$ years of age. These questions concerned those who framed the PCORI legislation, and so QALYs were essentially prohibited from our toolbox.

But that is very different from saying that we can't look at costs - or service utilization - as possible outcomes. We could look, for instance, at the number of days spent in the hospital with treatment A versus treatment B, which is an indirect measure of cost. Or out-of-pocket costs might be another meaningful outcome to use.

Q What is your timeline for moving forward? The draft of our methodology report which will assess the current best practices in CER and PCOR - will be completed and posted for public input in May 2012. And in September 2011 our first funding announcement will be released. This will be for pilot studies, and will have a methodological tone: it will be about assessing the potential of known and novel approaches to PCOR. It could, for example, fund studies that would identify and evaluate new methods for engaging patients and other stakeholders in the research process.

The primary CER and PCOR that we aim to do will have to wait until we've set our national priorities and articulated a research agenda, which I'd say you can look out for in mid-2012.

\section{Q How do you see the drug development} industry participating with the PCORI? I see a role for industry in generating questions. We also intend to work with them in the hopes of refining patient-centred methods for the research they use and we use. But I don't really see an industry organization applying to us for funding: most of the questions we prioritize and the studies we commission will be carried out through grants and contracts that will be awarded to clinicians and patient groups across the country, and will probably focus on post-approval products.

Q Given the increasing uncertainty over drug reimbursement, and a lack of real-world data at the time of approval that may slow uptake, is there perhaps a role for the PCORI in implementing CER into pre-approval clinical development as well?

As yet the PCORI board has not really addressed this question. But I would not rule out the possibility completely. With regard to non-standard trials, for example, we're certainly interested in methodology and might be able to make a contribution there. There is also some thought that we may be able to work with the US Food and Drug Administration to incorporate pre-approval studies into our analyses. 